\title{
RIGOROUS STRIP ADJUSTMENT OF AIRBORNE LASERSCANNING DATA BASED ON THE ICP ALGORITHM
}

\author{
P. Glira ${ }^{\mathrm{a} *}$, N. Pfeifer ${ }^{\mathrm{a}}$, C. Briese ${ }^{\mathrm{a}}$, C. Ressl ${ }^{\mathrm{a}}$ \\ ${ }^{a}$ Vienna University of Technology, Department of Geodesy and Geoinformation, Research Group Photogrammetry \\ (philipp.glira, norbert.pfeifer, christian.briese, camillo.ressl)@geo.tuwien.ac.at
}

Commission III, WG III/2

KEY WORDS: orientation, calibration, georeferencing, Iterative Closest Point algorithm

\begin{abstract}
:
Airborne Laser Scanning (ALS) is an efficient method for the acquisition of dense and accurate point clouds over extended areas. To ensure a gapless coverage of the area, point clouds are collected strip wise with a considerable overlap. The redundant information contained in these overlap areas can be used, together with ground-truth data, to re-calibrate the ALS system and to compensate for systematic measurement errors. This process, usually denoted as strip adjustment, leads to an improved georeferencing of the ALS strips, or in other words, to a higher data quality of the acquired point clouds. We present a fully automatic strip adjustment method that (a) uses the original scanner and trajectory measurements, (b) performs an on-the-job calibration of the entire ALS multisensor system, and (c) corrects the trajectory errors individually for each strip. Like in the Iterative Closest Point (ICP) algorithm, correspondences are established iteratively and directly between points of overlapping ALS strips (avoiding a time-consuming segmentation and/or interpolation of the point clouds). The suitability of the method for large amounts of data is demonstrated on the basis of an ALS block consisting of 103 strips.
\end{abstract}

\section{INTRODUCTION}

The main components of an ALS system are a laser scanner, a Global Navigation Satellite System (GNSS), and an Inertial Navigation System (INS). The GNSS and INS measurements serve to estimate the aircraft's trajectory, i.e. its position (three coordinates) and its orientation (three angles). Using the trajectory, the mounting calibration parameters (which describe the positional and rotational offset between the scanner and the GNSS/INS system), and the measurements of the scanner (range and angle(s)), the $3 \mathrm{~d}$ coordinates of the surface points can be determined. Systematic errors in any of these data inputs lead to a systematic, usually nonlinear deformation of the strip wise collected point clouds. These errors can be recognized in two forms: as discrepancies between overlapping strips and as discrepancies between strips and ground-truth data, e.g. ground control points. Using strip adjustment, the quality of the ALS point clouds can be improved by simultaneously minimizing these discrepancies (Figure 1).

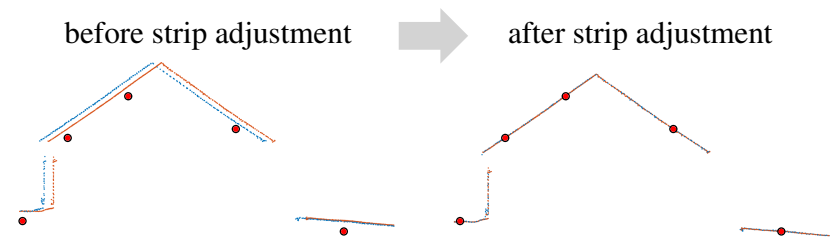

Figure 1: The discrepancies between overlapping strips (blue/orange) and between strips and ground control points (red) are minimized simultaneously by strip adjustment.

Parts of the strip adjustment method presented in this article are based on the Iterative Closest Point (ICP) algorithm (Besl and McKay (1992), Chen and Medioni (1992)). This algorithm is used to improve the alignment of two (or more) point clouds by minimizing iteratively the discrepancies within the overlap area

\footnotetext{
${ }^{*}$ Corresponding author
}

of these point clouds. Nowadays the term ICP does not necessarily refer to the algorithm presented in the original publications, but rather to a group of surface matching algorithms which have in common the following aspects:

I : correspondences are established iteratively

C : as correspondence the closest point, or more generally, the corresponding point, is used

$\mathbf{P}$ : correspondences are established on a point basis.

The key features of the presented strip adjustment method are:

Calibration of the ALS multisensor system The scanner's interior calibration and the mounting calibration are re-estimated in the strip adjustment. For this, additional calibration parameters are introduced into the general formulation of the direct georeferencing equation. This approach is rigorous, as the original scanner measurements and the trajectory of the aircraft are used as inputs.

Correction of the aircraft's trajectory The trajectory of the aircraft is considered to be estimated in advance from the GNSS and INS measurements (e.g. using some sort of Kalman filter (Hebel and Stilla, 2012)). As these measurements are affected by external influences (e.g. number of satellites), their accuracy is not constant, but dynamically changing. In order to reduce any possible systematic errors, trajectory correction parameters are estimated for each ALS strip individually.

Point based correspondences In order to exploit the full resolution of the data, correspondences are established on the basis of the original ALS points. A single correspondence is defined by two points and their normal vectors. In the Least Squares Adjustment, the point-to-plane distances of all correspondences are minimized simultaneously.

Iterative estimation Correspondences are not only established once, but iteratively. For each new set of correspondences, the parameters are re-estimated trough Least Squares Adjustment. This 
iterative procedure is repeated until convergence is reached, i.e. there are no significant changes in the estimated parameters.

Moreover, the presented method is suitable for large amounts of data (up to a few hundreds of strips), is fully automatic, and has no restriction on the object space in terms of shape. If available, ground-truth data can be considered.

The rest of the paper is organized as follows. After a review of related literature in section 2., the general formulation of the direct georeferencing process and its refinement with additional parameters is presented in section 3. On this basis, the strip adjustment method is presented in section 4. This includes a detailed description of the correspondences and the functional and stochastic model of the adjustment. In section 5., the strip adjustment method is demonstrated on the basis of an ALS block consisting of 103 strips. The conclusions are presented in the last section.

\section{RELATED WORK}

Basically, two types of strip adjustment methods exist: (a) rubbersheeting coregistration solutions, which use the measured terrain points only as input (Ressl et al., n.d.) and (b) rigorous solutions that start from the original scanner and trajectory measurements. Rigorous solutions, as the one presented in this article, differ mainly in terms of the estimated parameters and the used correspondences. Many methods concentrate on the estimation of the misalignment between the scanner and the INS (Hebel and Stilla, 2012; Toth, 2002). The most extensive parameter models are presented in Kager (2004) and Friess (n.d.). Correspondences are either generated on the basis of the original point cloud or a derivate of it (e.g interpolated grids or triangulations). Most approaches use planes as corresponding geometric elements. They can be of fixed size or variable size found by segmentation (e.g. rooftops). Kersting et al. (2012) use higher order primitives as correspondences. An overview of strip adjustment methods is presented in Toth (2009) and Habib and Rens (2007).

\section{DIRECT GEOREFERENCING}

\subsection{General formulation}

The direct georeferencing of ALS strips requires three data inputs: the scanner measurements, the trajectory of the aircraft, and the mounting calibration parameters (Skaloud and Lichti, 2006; Hebel and Stilla, 2012). Combining all these measurements, the point coordinates at time $t$ are given by

$$
\mathbf{x}^{e}(t)=\mathbf{g}^{e}(t)+R_{n}^{e}(t) R_{i}^{n}(t)\left(\mathbf{a}^{i}+R_{s}^{i} \mathbf{x}^{s}(t)\right)
$$

In this representation the superscript of a vector denotes the coordinate system in which it is defined, whereas the notation $R_{\text {source }}^{\text {target }}$ is used to denote a transformation from a source coordinate system to a target coordinate system. Consequently, four coordinate systems appear in equation (1):

$$
\begin{array}{ll}
s \text {-system } & \begin{array}{l}
\text { scanner coordinate system } \\
i \text {-system }
\end{array} \\
\begin{array}{l}
\text { INS coordinate system, sometimes also denoted as } \\
\text { body coordinate system } \\
\text { navigation coordinate system, equal to a local- } \\
\text { level coordinate system }
\end{array} \\
e \text {-system } & \begin{array}{l}
\text { Earth-Centered, Earth-Fixed (ECEF) coordinate } \\
\text { system }
\end{array}
\end{array}
$$

Definitions of these coordinate systems can be found in Bäumker and Heimes (n.d.). Furthermore, equation (1) includes: $\mathbf{x}^{s}(t) \quad 3$-by-1 vector with the coordinates of the laser point in the $s$-system. Generally, these coordinates can be expressed as a function of the range $\rho$ and two angles $\alpha$ and $\beta$ :

$$
\mathbf{x}^{s}(t)=\mathbf{x}^{s}(\rho(t), \alpha(t), \beta(t))
$$

$R_{s}^{i} \quad 3$-by-3 rotation matrix describing the rotation from the $s$-system to the $i$-system, i.e. from the scanner to the INS. This rotation is usually denoted as (boresight) misalignment and is parametrized trough three Euler angles:

$$
R_{s}^{i}=R_{s}^{i}(\omega, \phi, \kappa)
$$

$\mathbf{a}^{i}$ 3-by-1 vector describing the positional offset between the GNSS antenna and the origin of the $s$-system. This vector is usually denoted as lever arm.

$R_{i}^{n}(t)$ 3-by-3 rotation matrix describing the rotation from the $i$-system to the $n$-system as part of the trajectory data. This rotation can be estimated from the GNSS/INS measurements and is parametrized trough three Euler angles roll $\phi$, pitch $\theta$, and yaw $\psi$ :

$$
R_{i}^{n}(t)=R_{i}^{n}(\phi(t), \theta(t), \psi(t))
$$

$R_{n}^{e}(t)$ 3-by-3 rotation matrix describing the rotation from the $n$-system to the $e$-system. This rotation is not observed, but is a function of the longitude $\lambda$ and latitude $\varphi$ corresponding to the actual value of $\mathbf{g}^{e}(t)$ :

$$
R_{n}^{e}(t)=R_{n}^{e}(\lambda(t), \varphi(t))
$$

$\mathbf{g}^{e}(t) \quad 3$-by-1 vector describing the position of the GNSS antenna in the $e$-system as part of the trajectory data.

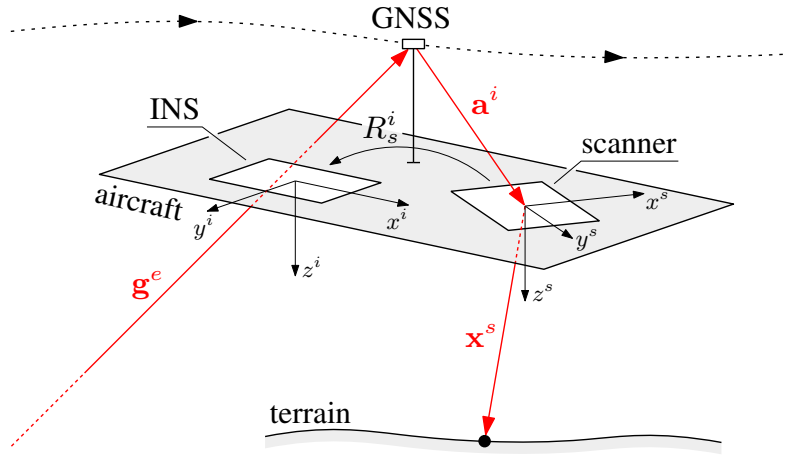

Figure 2: Direct georeferencing of ALS point clouds.

It should be noted, that the strip adjustment method presented herein is fully performed in the $e$-system. Only afterwards, the points obtained by equation (1) are projected from the $e$-system to an arbitrary mapping coordinate system ( $m$-system), e.g. UTM. This has the main advantage that the surface distortions applied in the $m$-system have not to be considered in the strip adjustment.

Side note If the trajectory is provided by an external company, in most of the cases it already relates to the $s$-system (in contrast to the definition given above). In this case

- the angles roll $\phi$, pitch $\theta$, and yaw $\psi$ directly describe the rotation from the $s$-system to the $n$-system:

$$
R_{s}^{n}(t)=R_{i}^{n}(t) R_{s}^{i}=R_{s}^{n}(\phi(t), \theta(t), \psi(t))
$$

- $\mathbf{g}^{e}(t)$ relates to the origin of the $s$-system, 
- and the lever arm $\mathbf{a}^{i}$ can be omitted in equation (1).

As a result, equation (1) simplifies to:

$$
\mathbf{x}^{e}(t)=\mathbf{g}^{e}(t)+R_{n}^{e}(t) R_{s}^{n}(t) \mathbf{x}^{s}(t)
$$

\subsection{Additional parameters}

Any measurement in equation (1) can be affected by systematic errors, which in turn cause a systematic (nonlinear) deformation of the ALS strip (Glennie, 2007; Habib and Rens, 2007). To minimize these errors within the strip adjustment, additional calibration and correction parameters have to be introduced into the direct georeferencing equation (1). These parameters can be divided into three groups:

Scanner calibration parameters The scanner calibration parameters compensate for the systematic errors of the ALS scanner's measurements $\mathbf{x}^{s}$. The estimation of these parameters within the adjustment is usually denoted as on-the-job calibration. A comprehensive analysis of scanner related errors and their causes can be found in Katzenbeisser (n.d.). The specific choice of parameters primarily depends on the construction type of the ALS scanner, especially on its beam deflection mechanism. For example, the parameters required to appropriately model the errors of scanners that deflect the laser beam only in one direction (linear scanners), differ from those that deflect the laser beam in a circular pattern (nutating scanners). Hence, no general recommendation can be given. Instead, we propose a calibration model which is universally applicable, although it may not be the optimal choice in any case. Therefore we formulate $\mathbf{x}^{s}$, according to equation (2), as a function of the polar coordinates $\rho, \alpha$, and $\beta$ :

$$
\mathbf{x}^{s}(t)=\left[\begin{array}{c}
\rho(t) \\
\cos \alpha(t) \sin \beta(t) \\
\rho(t) \sin \alpha(t) \\
\rho(t) \cos \alpha(t) \cos \beta(t)
\end{array}\right]^{s}
$$

For each polar coordinate two calibration parameters are introduced, an offset and a scale. This yields to three offset parameters $(\Delta \rho, \Delta \alpha, \Delta \beta)$ and three scale parameters $\left(\varepsilon_{\rho}, \varepsilon_{\alpha}, \varepsilon_{\beta}\right)$ which are defined by

$$
\begin{aligned}
& \rho(t)=\Delta \rho+\rho_{0}(t) \cdot\left(1+\varepsilon_{\rho}\right) \\
& \alpha(t)=\Delta \alpha+\alpha_{0}(t) \cdot\left(1+\varepsilon_{\alpha}\right) \\
& \beta(t)=\Delta \beta+\beta_{0}(t) \cdot\left(1+\varepsilon_{\beta}\right)
\end{aligned}
$$

where the original scanners's measurements are denoted by $\rho_{0}$, $\alpha_{0}$, and $\beta_{0}$.

EXAMPLE Linear scanners deflect the laser beam only in one direction (usually across the flight track). Thus, $\alpha$ can be interpreted as the beam deflection angle, whereas $\beta$ is equal to zero. The parameters associated with $\beta$, i.e. $\Delta \beta$ and $\varepsilon_{\beta}$, can be omitted in this case. The remaining parameters compensate for a range finder offset error $(\Delta \rho)$, a range finder scale error $\left(\varepsilon_{\rho}\right)$, a zeropoint error of the angular encoder $(\Delta \alpha)$, and a scale error of the angular encoder $\left(\varepsilon_{\alpha}\right)$. However, these parameters may also compensate for other correlated (and possibly unknown) effects. For example, the parameter $\varepsilon_{\alpha}$ not only serves to correct an angular scale error, but also minimizes the influence of the atmospheric refraction. Similarly, the parameter $\varepsilon_{\rho}$, which primarily corrects a range finder scale error, may also compensate range errors caused by the atmospheric propagation delay.

Trajectory correction parameters The trajectory of the aircraft, i.e. its orientation $R_{i}^{n}$ and its position $\mathbf{g}^{e}$, is estimated by the integration of GNSS and INS measurements in a Kalman filter. Skaloud et al. (2010) highlight the fact that all these measurements are strongly affected by external influences and hence their accuracy can not be assumed to be constant in time. As these systematic trajectory errors even vary within a single ALS strip, time dependent correction parameters should be estimated for each strip. However, the determinability of such parameters strongly depends on the terrain geometry and can not be guaranteed in any case (e.g. over flat terrain). We therefore limit the estimation on the constant part of the trajectory errors for each strip. For this an individual set of six trajectory correction parameters (three angle corrections and three position corrections) is assigned to each strip. The angle corrections $\left(\Delta \phi_{i}, \Delta \theta_{i}, \Delta \psi_{i}\right)$ for strip $i$ are defined by

$$
\begin{aligned}
\phi(t) & =\phi_{0}(t)+\Delta \phi_{i} \\
\theta(t) & =\theta_{0}(t)+\Delta \theta_{i} \\
\psi(t) & =\psi_{0}(t)+\Delta \psi_{i}
\end{aligned}
$$

where $\phi_{0}, \theta_{0}$, and $\psi_{0}$ denote the original values of the roll, pitch, and yaw angles. Accordingly, the position corrections $\left(\Delta g_{x i}\right.$, $\left.\Delta g_{y i}, \Delta g_{z i}\right)$ for strip $i$ are defined by

$$
\mathbf{g}^{e}(t)=\mathbf{g}_{0}^{e}(t)+\Delta \mathbf{g}^{e}=\left[\begin{array}{l}
g_{x 0}(t)+\Delta g_{x i} \\
g_{y 0}(t)+\Delta g_{y i} \\
g_{z 0}(t)+\Delta g_{z i}
\end{array}\right]^{e}
$$

where $g_{x 0}, g_{y 0}$, and $g_{z 0}$ denote the original position values.

Mounting calibration parameters In most of the cases the mounting calibration parameters, that is the misalignment $R_{s}^{i}$ and the lever-arm $\mathbf{a}^{i}$, are already known in advance, e.g. from a previously performed calibration or from construction plans of the system. However, these values can be inaccurate or outdated. Thus, it is recommended to re-estimate the mounting calibration by strip adjustment. Especially an incorrect misalignment, which is difficult to measure by terrestrial measurements, can cause very large point displacements, because the effect of angular errors is directly proportional to the object distance $\rho$. For this reason many strip adjustment methods concentrate on the estimation of $R_{s}^{i}$, neglecting other parameters (e.g. Toth (2002), Hebel and Stilla (2012)). The mounting calibration parameters are already included in equation (1), where the misalignment $R_{s}^{i}$ is defined by the angles $\omega, \phi, \kappa$, and the lever-arm is defined by the components of $\mathbf{a}^{i}$, namely $a_{x}, a_{y}$, and $a_{z}$.

Summarizing, the following parameters can be estimated within the proposed strip adjustment:

\section{- Scanner calibration parameters}

- range and angle offsets $\Delta \rho, \Delta \alpha, \Delta \beta$

- range and angle scales $\varepsilon_{\rho}, \varepsilon_{\alpha}, \varepsilon_{\beta}$

- Trajectory correction parameters (for each strip $i$ )

- angle corrections $\Delta \phi_{i}, \Delta \theta_{i}, \Delta \psi_{i}$

- position corrections $\Delta g_{x i}, \Delta g_{y i}, \Delta g_{z i}$

- Mounting calibration parameters

- misalignment angles $\omega, \phi, \kappa$

- lever-arm components $a_{x}, a_{y}, a_{z}$

This gives a total number of $12+6 n$ parameters for an ALS block consisting of $n$ strips. It should be noted that depending on the assembly of the sensors, the flight configuration, or the terrain geometry, some of these parameters may be completely correlated and therefore not estimable. The next section demonstrates how the parameters are estimated by strip adjustment. 


\section{STRIP ADJUSTMENT}

The main steps of the strip adjustment method are shown in Figure 3. As it can be seen, two iteration loops exist: within the outer iteration loop the correspondences are re-established (as in the ICP algorithm) after each run, whereas the inner iteration loop is needed to solve the non-linear equation system within the adjustment. In the following sections, the data inputs, the correspondences, and the adjustment are described in detail.

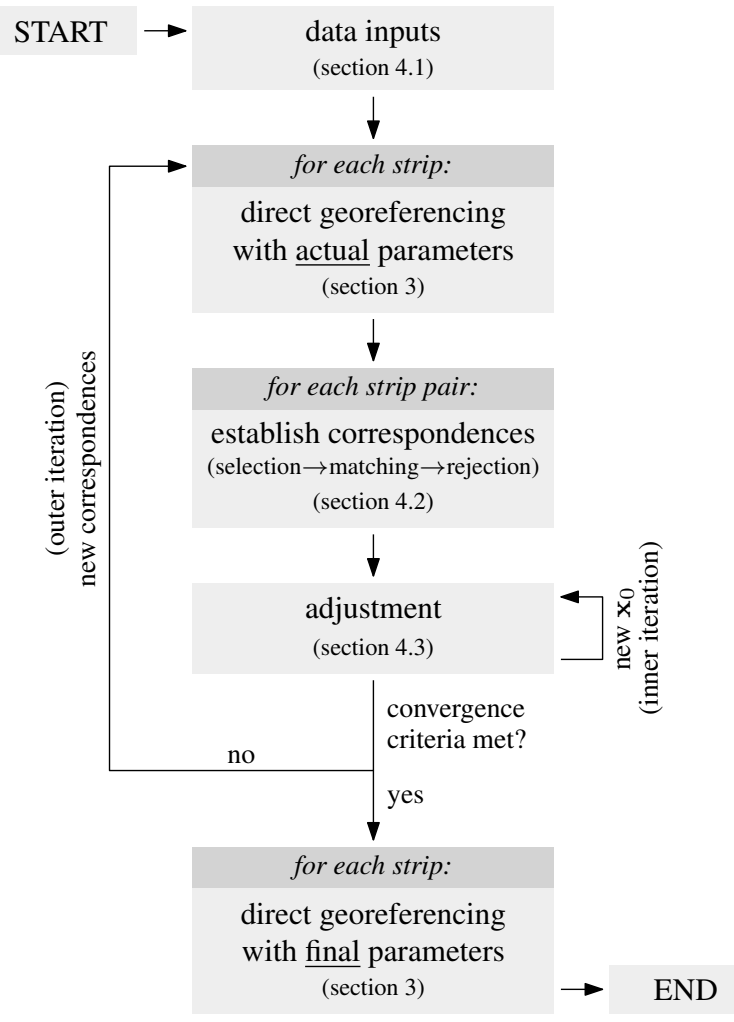

Figure 3: Flowchart of strip adjustment method.

\subsection{Data inputs}

The following data inputs are used in the strip adjustment:

- Scanner measurements: $\mathbf{x}^{s}(t)$

- Trajectory of the aircraft: $\mathbf{g}^{e}(t)$ and $R_{i}^{n}(t)$

- Mounting calibration parameters: $R_{s}^{i}$ and $\mathbf{a}^{i}$

These values are introduced as approximations in the Least Squares Adjustment. If no a priori information about the mounting calibration exists, then the misalignment $R_{s}^{i}$ should be chosen so that it approximately rotates the $s$-system into the $i$-system, whereas the lever-arm $\mathbf{a}^{i}$ can be approximated by the null vector.

- Ground-truth data (optional, see section 4.4)

\subsection{Correspondences}

As demonstrated in Glira et al. (2015), the strip adjustment results are strongly affected by the used correspondences. We propose to establish the correspondences on the basis of the original ALS points; the main reasons for this are: the highest possible resolution level of the data is exploited, no time-consuming preprocessing of the data is required (in contrast to correspondences which are found by segmentation and/or interpolation), and no restrictions are imposed on the object space (e.g. the presence of rooftops or horizontal fields). A single correspondence is defined by two points from overlapping strips and their normal vectors (estimated from the neighbouring points). As a point and its normal vector define a tangent plane, consequently, a correspondence represents two homologous tangent planes in object space. In the Least Squares Adjustment the so-called point-to-plane distance, which is defined as the perpendicular distance from one point to the tangent plane of the other point, is minimized.

The correspondences are established for each pair of overlapping strips in three distinct steps: the Selection, Matching, and Rejection step. A comprehensive description of these steps can be found in Glira et al. (2015). Thus, only a brief summary is provided here.

Selection In this step, a subset of points is selected within the overlap area in one strip. For this task four selection strategies are considered. The main difference between these strategies is the information used as input for the point selection; see Table 4.2. The four selection strategies, sorted by increasing computational complexity, are:

- Random Sampling (RS) This is the fastest strategy, because points are simply selected randomly, without considering the coordinates or the normal vectors of the points. For ALS point clouds, in which the point density is nearly constant, this option can be considered as an approximation of uniform sampling.

- Uniform Sampling (US) The aim of this strategy is to select points in object space as uniformly as possible. This leads to a homogeneous distribution of the selected points, where regions of equal area are equally weighted within the adjustment. On the contrary, if a normal direction is predominating (e.g. flat terrain), many redundant points with approximately parallel normal vectors, which do not contribute significantly to the parameter estimation, are selected. This option was implemented by dividing the overlap area into a voxel structure and selecting the closest point to each voxel center. Consequently, the edge length of a single voxel can be interpreted as the mean sampling distance along each coordinate direction.

- Normal Space Sampling (NSS) The aim of this strategy is to select points such that the distribution of their normals in angular space is as uniform as possible (Rusinkiewicz and Levoy, 2001). For this the angular space (slope vs. aspect) is divided into classes (e.g. $2.5^{\circ} \times 10^{\circ}$ ), and points are randomly sampled within these classes. This strategy does not consider the position of the points.

- Maximum Leverage Sampling (MLS) This strategy selects those points, which are best suited for the estimation of the parameters. For this, the effect of each point on the parameter estimation, i.e. its leverage, is considered. The points with the maximum leverage (= the lowest redundancy) are selected. This strategy considers the coordinates and the normal vectors of the points. Details on the algorithm can be found in Glira et al. (2015).

\begin{tabular}{l|cccc} 
& RS & US & NSS & MLS \\
\hline coordinates of points & no & yes & no & yes \\
normal vectors of points & no & no & yes & yes
\end{tabular}

Table 1: Information used by the selection strategies.

Figure 4 compares the selection strategies on the basis of a (in terms of orientation) rather difficult dataset. As it can be seen, this scene contains only one terrain feature - a ditch - which can constrain the transformation at its finest level. As RS and US don't consider the orientation of the normal vectors, many redundant points are selected on the flat terrain and only a few points are selected within the ditch. As a consequence, both strategies are only recommended for terrain with steady height variations 
(e.g. urban area, hilly or mountainous terrain; see example in section 5.). However, NSS considers the normal vectors and thus a sufficient number of points is selected within the ditch. MLS additionally evaluates the coordinates of the points in order to estimate the leverage of each point on the parameter estimation. As a result, points are primarily selected in the ditch and towards the edges of the overlap area.

Random Sampling (RS)

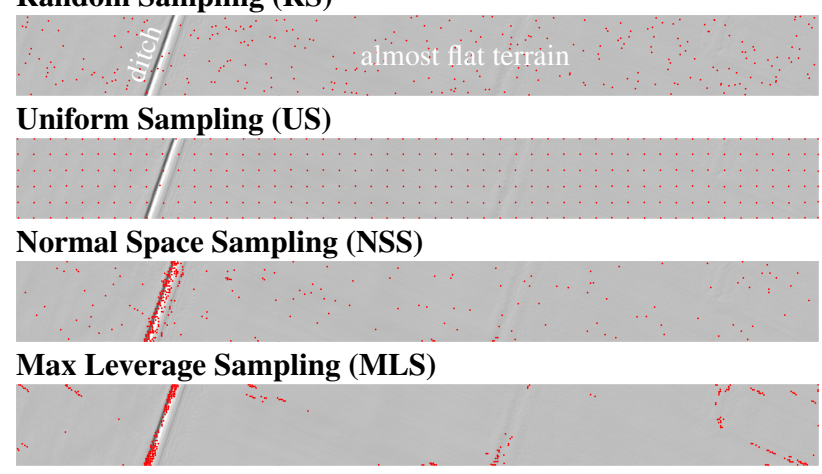

Figure 4: Comparison of correspondence selection strategies (from Glira et al. (2015)). Each strategy was applied to select approximately 300 points. A shaded Digital Elevation Model (DEM) of the ALS scene is visualized in the background.

Matching This step establishes the correspondences. For this, each selected point from the previous step is paired to the nearest neighbour (closest point) of the overlapping strip. As in the adjustment the point-to-plane distance is minimized for each correspondence, two associated points don't have to be identical in object space, but they only have to belong to the same (tangent) plane (e.g. flat terrain surface). Due to the good initial orientation and the high point density in ALS, this requirement is mostly fulfilled. The nearest neighbour search can be realized efficiently using k-d trees.

Rejection The aim of this step is the a priori identification and subsequent rejection of unreliable or false correspondences. Each correspondence is tested for three criteria:

- Rejection based on the plane roughness of corresponding points For the minimization of the point-to-plane distance, the reliability of both normal vectors must be ensured. Of course, this condition is not met if the scanned object can not be appropriately modelled by a plane, e.g. in the case of vegetation. This is directly reflected by the a posteriori reference variance $\hat{\sigma}_{0}^{2}$ of the tangent plane adjustment. We denote the standard deviation $\hat{\sigma}_{0}$ as the plane roughness and reject all correspondences where at least one of the two $\hat{\sigma}_{0}$ values exceeds an upper limit $\sigma_{\max }$. We usually set $\sigma_{\max }=$ $10 \mathrm{~cm}$.

- Rejection based on the angle between the normal vectors of corresponding points To ensure that two corresponding points belong to the same plane, the angle $\alpha$ between the normal vectors of these points should not exceed an upper limit $\alpha_{\max }$. We usually set $\alpha_{\max }=5^{\circ}$.

- Rejection based on the point-to-plane distance between corresponding points Apart from a few false correspondences, the a priori point-to-plane distances $d_{1}, d_{2}, \ldots, d_{n}$ are assumed to be normally distributed for each individual pair of overlapping strips. A robust estimator for the standard deviation of this contaminated set of correspondences is given by

$$
\sigma_{\operatorname{mad}}=1.4826 \cdot \mathrm{mad}
$$

where mad is the median of the absolute differences (with respect to the median) (Hampel, 1974). In this work, all correspondences with a point-to-plane distance outside the range of $\tilde{d} \pm 3 \sigma_{\text {mad }}$ are rejected, where $\tilde{d}$ denotes the median of the point-to-plane distances of all correspondences that passed the first two criteria.

It is not guaranteed that all false correspondences are rejected by this three criteria. Thus, a robust adjustment method is used for the detection and removal of the remaining ones.

\subsection{Adjustment}

A robust Least Squares Adjustment is performed in order to estimate the parameters described in section 3.2. The objective of the adjustment is to minimize the weighted sum of squared pointto-plane distances

$$
\Omega=\sum_{i=1}^{n}\left(w_{i} d_{i}^{2}\right) \rightarrow \text { minimum }
$$

where $w_{i}$ is the weight, and $d_{i}$ is the point-to-plane distance of the $i$-th correspondence. In the following, the definitions of $w_{i}$ (stochastic model) and $d_{i}$ (functional model) are given.

Definition of point-to-plane distance The signed point-to-plane distance $d_{i}$ (Figure 5) is defined as the perpendicular distance from a point to a plane. It is conveniently expressed by the Hessian normal form

$$
d_{i}=\left(\mathbf{p}_{i}-\mathbf{q}_{i}\right)^{T} \mathbf{n}_{i}
$$

where $\mathbf{p}_{i}$ and $\mathbf{q}_{i}$ are the corresponding points of the $i$-th correspondence, and $\mathbf{n}_{i}$ is the normal vector associated to the point $\mathbf{p}_{i}$ (with $\left\|\mathbf{n}_{i}\right\|=1$ ). In this eq. the points $\mathbf{p}_{i}$ and $\mathbf{q}_{i}$ are determined by the direct georeferencing equation (1), i.e. in consideration of the scanner measurements, the trajectory, the mounting calibration parameters, and the additional parameters described in section 3.2.

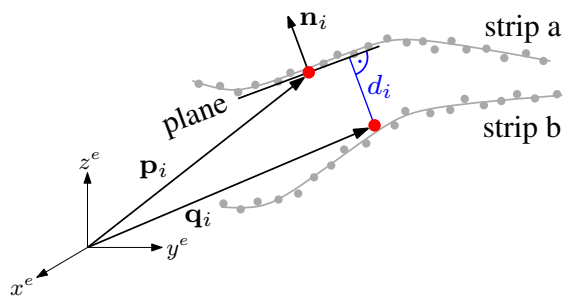

Figure 5: The point-to-plane distance $d_{i}$ for the $i$-th correspondence is defined as the perpendicular distance from point $\mathbf{q}_{i}$ to the tangent plane defined by $\mathbf{p}_{i}$ and $\mathbf{n}_{i}$. The point $\mathbf{q}_{i}$ is the nearest neighbour of the point $\mathbf{p}_{i}$.

Definition of weights The weights of the correspondences $w_{i}$ could be estimated in a mathematically rigorous way by propagating the errors of the original measurements on the point-toplane distances $d_{i}$. For that, the influencing factors (especially on the range measurement) need to be considered, e.g. the precision of the scanner and trajectory measurements, the precision of the mounting calibration parameters, the roughness of the ground, the material of the ground (especially its porosity and its water content), the incidence angle, the beam divergence, and the energy distribution within the footprint. In order to model these influences, many inputs are required, from which some parts may not be available or stem from unreliable sources. Instead, we propose to estimate the precision of the point-to-plane distances iteratively (i.e. before each adjustment) from the point clouds itself or, more specifically, from the previously established correspondences. Let us assume that the correspondence $i$ belongs to the 
strip pair $k$, then its weight is determined by

$$
w_{i}=\frac{1}{\sigma_{k}^{2}}
$$

where $\sigma_{k}$ is the $\sigma_{\text {mad }}$ value (16) of all (non-rejected) point-toplane distances belonging to the strip pair $k$.

Adjustment solution The functional model of the adjustment evolves from the condition, that for each pair of corresponding points $\left(\mathbf{p}_{i}, \mathbf{q}_{i}\right)$, the point $\mathbf{q}_{i}$ should be in the tangent plane of point $\mathbf{p}_{i}$. In other words, the point-to-plane distances $d_{i}$ (cf. eq. (18)) should be equal to zero for all $n$ correspondences. This can be formulated for each individual correspondence by writing

$$
d_{i}=0+v_{i} \quad \text { for } i=1, \ldots, n
$$

where $v_{i}$ denotes the residual distance after adjustment. From these $n$ equations, the $u$-dimensional parameter vector $\mathbf{x}$ is estimated in consideration of the objective function stated in (17). For this, we rewrite the equation system (20) in vector notation, whereby the point-to-plane distances $d_{i}$ are expressed as a function of the estimated parameters $\hat{\mathbf{x}}$

$$
f(\hat{\mathbf{x}})=\mathbf{v}
$$

In order to resolve this equation system for the parameters $\hat{\mathbf{x}}$, the nonlinear functions $f(\hat{\mathbf{x}})$ must be linearized by

$$
f(\hat{\mathbf{x}})=f\left(\mathbf{x}_{0}\right)+\left.\frac{\partial f(\mathbf{x})}{\partial \mathbf{x}}\right|_{\mathbf{x}_{=} \mathbf{x}_{0}} \cdot \Delta \hat{\mathbf{x}}=f\left(\mathbf{x}_{0}\right)+A \Delta \hat{\mathbf{x}}
$$

where $\mathbf{x}_{0}$ denotes the vector of approximate parameter values, and $A$ denotes the $n$-by- $u$ design matrix which includes the partial derivatives of the equations with respect to the parameters at the point $\mathbf{x}_{0}$. The parameter estimates $\hat{\mathbf{x}}$ and the residuals $\mathbf{v}$ can then be determined by the equations

$$
\begin{aligned}
& \hat{\mathbf{x}}=\mathbf{x}_{0}+\Delta \hat{\mathbf{x}} \\
& \mathbf{v}=A \Delta \hat{\mathbf{x}}
\end{aligned}
$$

with

$$
\Delta \hat{\mathbf{x}}=\left(A^{T} P A\right)^{-1} A^{T} P\left(-f\left(\mathbf{x}_{0}\right)\right)
$$

and

$$
P=\operatorname{diag}\left(w_{1}, \ldots, w_{n}\right)
$$

The iterative process of relinearization terminates when $\Delta \hat{\mathbf{x}}$ becomes insignificantly different to zero (inner iteration, cf. Figure 3). We use the termination criterion described in (Kraus, 1997, p. 76).

In order to identify wrong correspondences (outliers), a $l_{1}$-norm minimization is imitated within the adjustment by iteratively reweighting the observations (Kraus, 1997, p. 218). After the removal of the detected outliers, a final regular Least Squares Adjustment is performed with the remaining correspondences (inliers). The amount of outliers strongly depends on the input data, but typically does not exceed $5 \%$.

\subsection{Datum definition and ground-truth data}

The datum of the ALS block can be defined in two ways: (a) by fixing the trajectory of one or more strips (i.e. by omitting the trajectory correction parameters of these strips) or (b) by considering ground-truth data (if available). Ground-truth data can be provided in many forms, e.g. as single (widely isolated) points from total station or GNSS measurements, as georeferenced point clouds from Terrestrial Laser Scanning (TLS), or as a DEM to which the ALS block should fit. Thus, a flexible concept, that can handle all these possibilities, is needed. We propose to treat the various forms of ground-truth data simply as further point clouds, whose orientations are fixed in object space. Additional correspondences between ground-truth data and overlapping ALS strips are introduced by equation (18), whereby the ground-truth data is represented by the points $\mathbf{q}_{i}$. Using $\mathbf{q}_{i}$ (instead of $\mathbf{p}_{i}$ ) for the ground-truth data has the major advantage, that the normals $\mathbf{n}_{i}$ are estimated from the ALS points, and thus also isolated points (with unknown normal vector) can be used as ground-truth data.

\section{EXPERIMENTAL RESULTS}

The presented strip adjustment method was applied to an ALS block located in the Austrian Alps (Tyrol, Kaunertal, Gepatschferner). The block consists of 95 longitudinal strips and 8 cross strips (Figure 6 (a)); the cross strips were flown in both directions. The ALS system was carried by a helicopter that flew over the terrain in a constant height above ground of approx. $600 \mathrm{~m}$. Further information about the flight campaign is summarized in Table 5.. A quality control of the delivered data revealed large systematic discrepancies (of up to several $\mathrm{dm}$ ) in the overlap area of neighbouring strips. For this reason, an improvement of the georeferencing of the data by means of strip adjustment, was absolutely necessary.

$\begin{array}{ll}\text { scanner model } & \begin{array}{l}\text { Riegl LMS-Q680i } \\ \text { approx. } 119 \mathrm{~km}^{2}\end{array} \\ \text { area of ALS block } & 103 \\ \text { no. of strips } & 4 \\ \text { no. of ground-truth data areas } & 58.8 \mathrm{~GB} \\ \text { size of input data } & 1455111768 \\ \text { overall no. of points } & 2.5 \text { points } / \mathrm{m}^{2} \\ \text { mean point density (single strip) } & 12.2 \text { points } / \mathrm{m}^{2} \\ \text { mean point density (block) } & 256 \mathrm{~Hz} \\ \text { frequency of trajectory data } & 2100 \text { to } 3450 \mathrm{~m} \\ \text { terrain elevation } & \end{array}$

Table 2: Key parameters of the ALS data used.

Next to the georeferenced strips, also the original scanner measurements and the trajectory data were provided. However, no a priori information about the mounting calibration was available. Thus, approximate values for these parameters were determined as described in section 4.1. In total, 627 parameters were estimated in the adjustment; this includes 3 scanner calibration parameters (range offset $\Delta \rho$, range scale $\varepsilon_{\rho}$, scan angle scale $\left.\varepsilon_{\alpha}\right), 6$ mounting calibration parameters, and 6 trajectory correction parameters for each of the 103 strips. As it can be seen in Figure 6 (b), five correspondence iterations (cf. Figure 3) were performed within the strip adjustment, although three iterations would have been sufficient. The reason for this is that we used, instead of a convergence criteria based e.g. on the change of the parameter estimates $\hat{\mathbf{x}}$ after each iteration, a fixed number of 5 correspondence iterations.

In total, 748 pairs of overlapping strips were identified. The ("strip to strip") correspondences between each of these strip pairs were selected by the uniform sampling strategy (mean sampling distance $=50 \mathrm{~m}$ ). In this mountainous region, this strategy led to a broad and relatively homogeneous distribution of the normal vector directions. The normal vectors were estimated by considering all neighbouring points within a fixed search radius of $2 \mathrm{~m}$. In order to ensure the reliability of the normal vector estimation, correspondences were rejected within rough surface areas (e.g. vegetation) by choosing $\sigma_{\max }=10 \mathrm{~cm}$. The positions of the correspondences are visualized in Figure 6 (c). The distribution of the a posteriori point-to-plane distances $d_{i}$ (equal to the residuals $v_{i}$, see eq. (20)) is shown in Figure 6 (d). The residuals are 
(a) ALS block: 103 strips and 4 ground-truth data areas

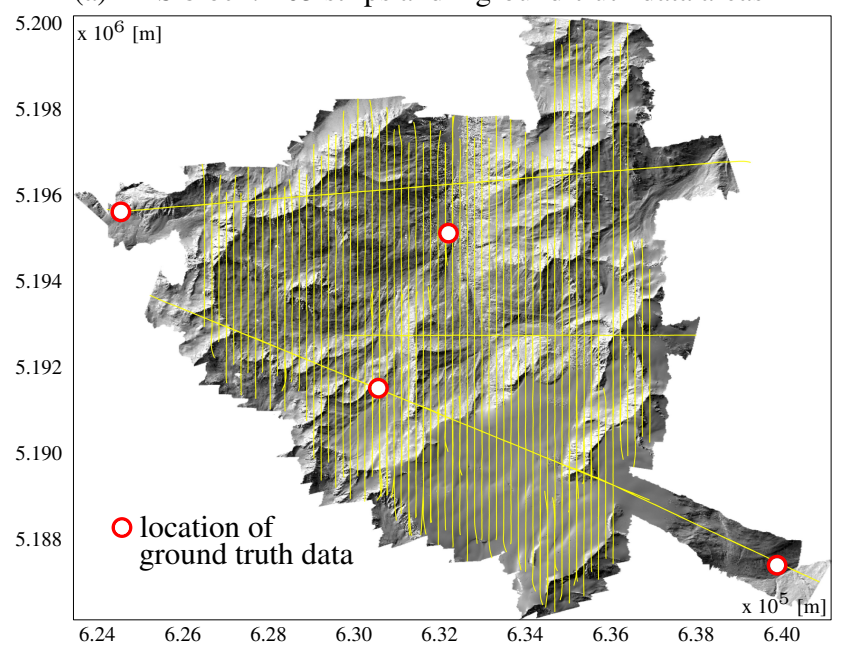

(b) Convergence of std.dev. of residuals over iterations

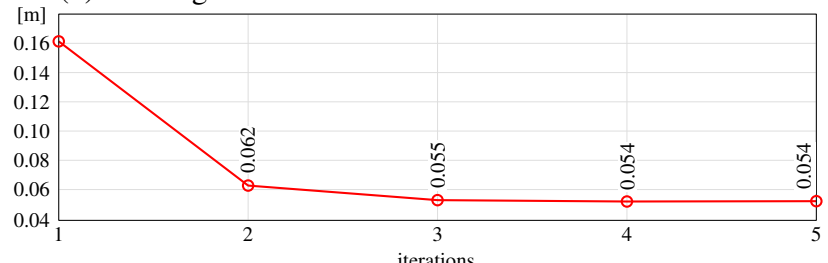

(c) Position of the correspondences (total number $=345205$ )

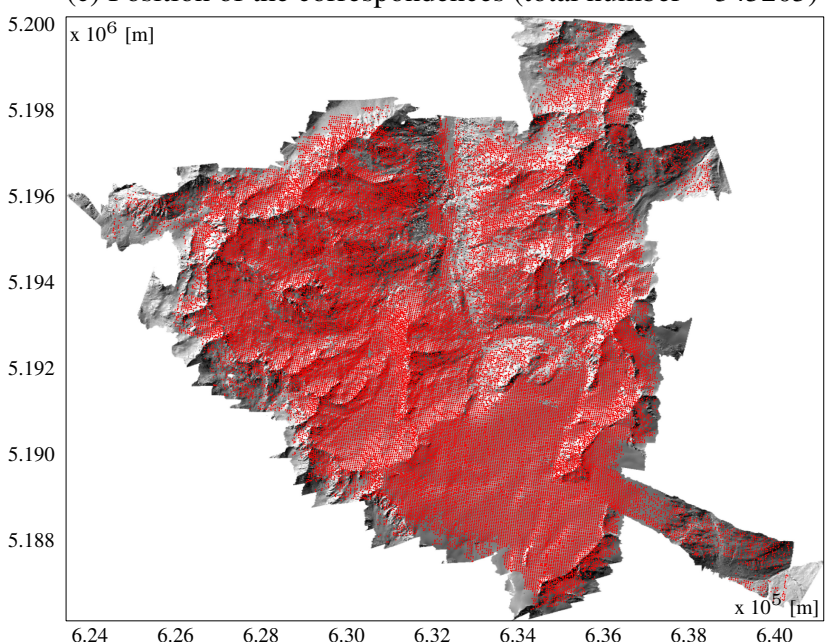

(d) Distribution of the a posteriori point-to-plane distances

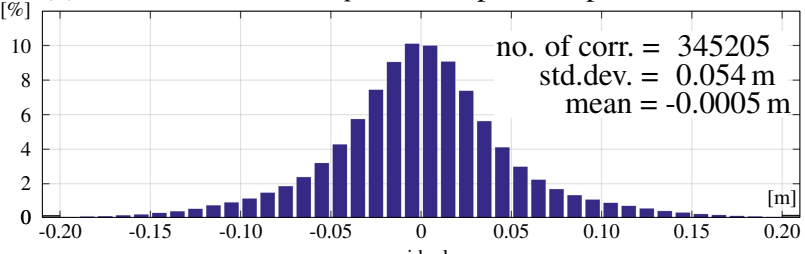

Figure 6: Strip adjustment results for an ALS block covering an area of approx. $119 \mathrm{~km}^{2}$ in the Austrian Alps (Kaunertal).

normally distributed, which indicates that systematic errors were widely eliminated by strip adjustment and confirms the appropriateness of the applied transformation model. In Figure 7 the mean and the standard deviation of these residuals are visualized individually for each strip pair. The strip pairs are thereby ordered by decreasing number of correspondences. As expected, the magnitude of the mean values is increasing by decreasing no. of correspondences (which is equivalent to a decreasing weight of the strip pairs), whereas the standard deviation remains, mainly due to the homogeneity of the terrain and of the surveying conditions, nearly constant.

The datum of the ALS block was defined by 4 ground-truth data areas (Figure 6 (a)). Altogether, these areas consist of 205 points, which were chosen predominantly on roofs and streets and were determined by a combination of static GNSS and total station measurements. These points were matched with 24 overlapping strips, giving in total 632 datum-defining ("ground-truth data to strip") correspondences.

no. of estimated parameters

no. of iterations

no. of overlapping strip pairs

748

no. of correspondences ("strip to strip") 345205

$\rightarrow$ mean of residuals

$-0.0005 \mathrm{~m}$

$\rightarrow$ std.dev. of residuals

$0.054 \mathrm{~m}$

no. of correspondences ("gtd to strip")

632

$\rightarrow$ mean of residuals

$-0.0002 \mathrm{~m}$

$\rightarrow$ std.dev. of residuals

$0.053 \mathrm{~m}$

Table 3: Strip adjustment results $($ gtd = ground-truth data).

\section{SUMMARY AND OUTLOOK}

We presented a new strip adjustment method which: (a) Number of correspondences for each strip pair

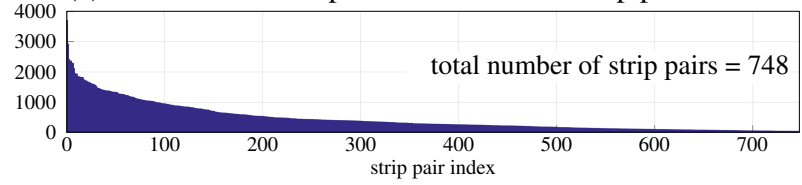

(b) Mean of a posteriori point-to-plane distances

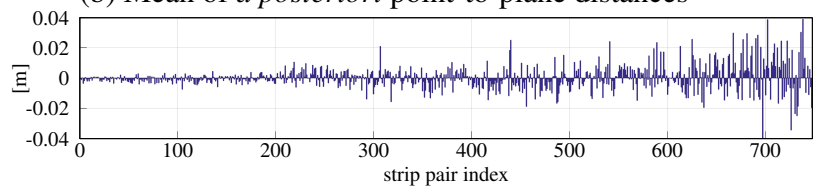

(c) Std. dev. of the a posteriori point-to-plane distances

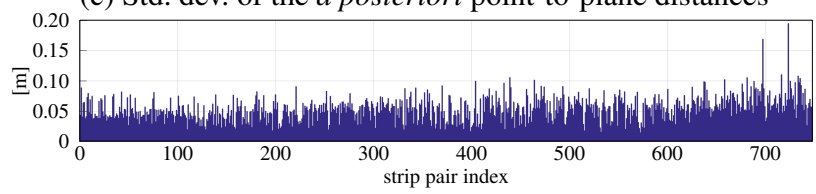

Figure 7: Strip adjustment results for all 748 overlapping strips pairs. The strip pairs are ordered according to the no. of correspondences.

- is formulated in a mathematically rigorous way, i.e. the original scanner measurements and the trajectory are used as data inputs

- estimates the ALS scanner calibration parameters

- estimates the mounting calibration of the ALS scanner

- corrects the aircraft's trajectory individually for each strip

- uses correspondences on the basis of the original ALS points

- establishes the correspondences not only once, but iteratively until convergence is reached

- minimizes simultaneously the point-to-plane distances of all correspondences

- can handle large amounts of data (up to a few hundreds of 
strips)

- is robust against false correspondences (outliers)

- considers ground-truth data in most forms, e.g. single ground control points or DEMs

- poses no restrictions on the object space in terms of shape (e.g. the presence of rooftops or horizontal fields)

- is fully automatic.

The suitability of the method for large amounts of data was demonstrated on the basis of a larger ALS block, in which systematic discrepancies were widely eliminated. Currently, we are working on the following extensions of the presented method:

1. Time-dependent trajectory correction parameters In rare cases, the trajectory measurements can be of very poor quality, i.e. the accuracy of the trajectory is highly variable, even within a single strip. In such cases, the trajectory correction parameters described in section 3.2 might not be sufficient, as they only compensate the errors on average for each strip. Thus, we plan to extend the parameter model by time-dependent trajectory correction parameters.

2. Synchronization error between scanner and trajectory As the scanner and trajectory measurements stem from different sensors, it may occur that they are not correctly synchronized (Katzenbeisser, n.d.). Such a synchronization error can cause discrepancies between overlapping strips in the order of a few $\mathrm{dm}$. This error can be estimated and corrected by means of strip adjustment.

3. RANSAC based rejection of false correspondences The rejection of unreliable or false correspondences is described in section 4.2. In order to further improve the detection of these outliers, we plan to additionally apply a new rejection method which is based on the RANSAC algorithm.

The strip adjustment method presented herein will be integrated into the software package OPALS (Pfeifer et al., 2014).

\section{ACKNOWLEDGEMENTS}

The authors would like to thank Mr. Helmuth Kager for his valuable contributions to this project. This research was carried out within the project PROSA (high-resolution measurements of morphodynamics in rapidly changing PROglacial Systems of the Alps) which is a joined project of the German research community (DFG) (BE 1118/27-1) and the Austrian Science Fund (FWF) (I 1647).

\section{References}

Bäumker, M. and Heimes, F., n.d. New calibration and computing method for direct georeferencing of image and scanner data using the position and angular data of an hybrid inertial navigation system. In: OEEPE Workshop, "Integrated Sensor Orientation", Hannover, Germany.

Besl, P. J. and McKay, N. D., 1992. Method for registration of 3-d shapes. In: Robotics-DL tentative, International Society for Optics and Photonics, pp. 586-606.

Chen, Y. and Medioni, G., 1992. Object modelling by registration of multiple range images. Image and Vision Computing 10(3), pp. 145 - 155. Range Image Understanding.

Friess, P., n.d. Toward a rigorous methodology for airborne laser mapping. In: Proceedings EuroCOW, Castelldefels, Spain.

Glennie, C., 2007. Rigorous 3d error analysis of kinematic scanning lidar systems. Journal of Applied Geodesy 1(3), pp. 147157.
Glira, P., Pfeifer, N., Ressl, C. and Briese, C., 2015. A correspondence framework for als strip adjustments based on variants of the ICP algorithm. Journal for Photogrammetry, Remote Sensing and Geoinformation Science (accepted).

Habib, A. and Rens, J., 2007. Quality assurance and quality control of lidar systems and derived data. In: Advanced Lidar Workshop, University of Northern Iowa, United States.

Hampel, F. R., 1974. The influence curve and its role in robust estimation. Journal of the American Statistical Association 69(346), pp. 383-393.

Hebel, M. and Stilla, U., 2012. Simultaneous calibration of ALS systems and alignment of multiview LiDAR scans of urban areas. Geoscience and Remote Sensing, IEEE Transactions on 50(6), pp. 2364-2379.

Kager, H., 2004. Discrepancies between overlapping laser scanner strips-simultaneous fitting of aerial laser scanner strips. International Archives of Photogrammetry, Remote Sensing and Spatial Information Sciences 35(B1), pp. 555-560.

Katzenbeisser, R., n.d. About the calibration of lidar sensors. In: ISPRS Workshop "3-D Reconstruction form Airborne LaserScanner and InSAR data”; 8-10 Oct. 2003, Dresden, Germany.

Kersting, A. P., Habib, A. F., Bang, K.-I. and Skaloud, J., 2012. Automated approach for rigorous light detection and ranging system calibration without preprocessing and strict terrain coverage requirements. Optical Engineering 51(7), pp. 076201-1.

Kraus, K., 1997. Photogrammetry, Vol.2, Advanced Methods and Applications. Duemmler / Bonn.

Pfeifer, N., Mandlburger, G., Otepka, J. and Karel, W., 2014. OPALS - A framework for airborne laser scanning data analysis. Computers, Environment and Urban Systems 45(0), pp. $125-136$.

Ressl, C., Pfeifer, N. and Mandlburger, G., n.d. Applying 3D affine transformation and least squares matching for airborne laser scanning strips adjustment without GNSS/IMU trajectory data. In: Proceedings of ISPRS Workshop Laser Scanning 2011, Calgary, Canada.

Rusinkiewicz, S. and Levoy, M., 2001. Efficient variants of the ICP algorithm. In: 3-D Digital Imaging and Modeling, 2001. Proceedings. Third International Conference on, Quebec City, Canada, pp. 145-152.

Skaloud, J. and Lichti, D., 2006. Rigorous approach to bore-sight self-calibration in airborne laser scanning. ISPRS journal of photogrammetry and remote sensing 61(1), pp. 47-59.

Skaloud, J., Schaer, P., Stebler, Y. and Tomé, P., 2010. Real-time registration of airborne laser data with sub-decimeter accuracy. ISPRS Journal of Photogrammetry and Remote Sensing 65(2), pp. 208-217.

Toth, C. K., 2002. Calibrating airborne lidar systems. In: ISPRS Commission II, Symposium 2002, Xian, China, ISPRS Archives, Volume XXXIV Part 2, pp. 475-480.

Toth, C. K., 2009. Strip adjustment and registration. In: Topographic Laser Ranging and Scanning-Principles and Processing (Ed.: Shan, J., Toth, C. K. / CRC Press), pp. 235-268. 